\title{
Utilidad de la precipitación obtenida por satélite en la modelación hidrológica aplicada a la cuenca del río Júcar
}

\author{
Usefulness of satellite-estimated rainfall in hydrologic modeling \\ of the Jucar river basin in spain
}

Lía Ramos Fernández¹; Félix Francés García ${ }^{2}$

\begin{abstract}
Resumen
Los actuales modelos hidrológicos distribuidos permiten simular caudales no únicamente en la salida de una cuenca, sino en cualquier parte de la misma, pero la eficacia de estos modelos depende de la disponibilidad de los datos de entrada. Es así que la lluvia estimada de satélite a escala global, se adapta a estos modelos distribuidos ya que se tienen datos de lluvia para toda la cuenca. Sin embargo, debido a la multidimensionalidad del error de la lluvia estimada de satélite, es difícil establecer a priori un producto que permita una óptima aplicación hidrológica en diferentes condiciones climáticas; es por eso que se hace necesario evaluar su desempeño a través de la modelación hidrológica. En este estudio, se evalúa la utilidad de la lluvia estimada por satélite a través de un modelo hidrológico lluvia-escorrentía y se emplea la lluvia estimada por el algoritmo PERSIANN a una resolución temporal diaria y resolución espacial de $0,25^{\circ}$ para el periodo comprendido entre el $1^{\circ}$ de marzo del 2000 al 31 de octubre del 2009 en la cuenca del río Júcar (España), obteniéndose resultados prometedores. Resulta el mejor rendimiento del modelo en calibración con valores de 0,384 y 0,499 del índice de Nash-Sutcliffe a la salida de las subcuencas Pajaroncillo y Sueca.
\end{abstract}

Palabras clave: lluvia estimada de satélite; PERSIANN; modelo hidrológico distribuido; río Júcar.

\begin{abstract}
The current hydrological models available allaw the simulation of output flow in the basin and also on any part of the basin, but the effectiveness of these models depends on the availability of input data. That is why the satellite-estimated rainfall on global scale adapts to these models, since the data has the rainfall information for the entire basin. However, due to the multidimensionality of error in satellite-estimated rainfall, it's difficult to establish beforehand a product which allows optimal hydrological application in different weather conditions. For this reason, it's necessary to evaluate its performance through hydrological modeling. This investigation analyses the usefulness of satellite-estimated rainfall through of a distributed hydrological model. Ground gauge rainfall were used to evaluate the algorithm PERSIANN with spatial resolution of $0,25^{\circ}$ and daily resolution for period length from March 01, 2000 to October 31, 2009 in the eastern Iberian Peninsula of Spain. We obtained promising results. The best performance was obtained in calibration of hydrological model with values of 0,384 and 0,499 of the Nash-Sutcliffe index to the output of the sub Pajaroncillo and Sueca.
\end{abstract}

Keywords: satellite-estimated rainfall; PERSIANN; distributed hydrological model; Jucar River.

\section{Introducción}

La modelización hidrológica tiene un papel importante en la mayoría de aspectos de la gestión del agua y del ambiente. Sin embargo, la eficacia de estos modelos depende de la disponibilidad de los datos de entrada. Así, los actuales modelos hidrológicos distribuidos requieren datos distribuidos espacialmente y con la precisión suficiente para permitir la investigación y aplicación. Durante las últimas décadas, la tecnología de sensores de satélite ha facilitado el desarrollo de enfoques innovadores para disponer en tiempo real de información meteorológica global. Biftu and Gan (2001) indican que frente a los datos convencionales que representan mediciones en un punto, las realizadas con sensores de satélite son promedios espaciales en los pixeles, y como tales, se ajustan a la necesidad de los modelos hidrológicos distribuidos, con una cobertura mundial, proporcionando información en regiones que son inaccesibles para otros sistemas de observación. En ese contexto, se han desarrollado algoritmos basados en satélites para

\footnotetext{
1 Departamento de Recursos Hídricos. Universidad Nacional Agraria La Molina, Lima, Perú. Email: liarf@lamolina.edu.pe

2 Institute of Water and Environmental Engineering. Universidad Técnica de Valencia, España. Email: ffrances@hma.upv.es
} 
producir estimaciones de variables climáticas como precipitación, temperatura, albedo, índice de área foliar, contenido de humedad de la superficie del suelo y nieve (Engman, 1995). Destacan los estudios para la estimación de la precipitación (Sorooshian et al., 2000); temperatura (Hansen et al., 2009); radiación (Andersen et al., 2002; Biftu y Gan, 2001) y evapotranspiración (Chen et al., 2005; Boegh et al., 2004). Estos algoritmos generan datos de alta resolución espacial y temporal con potencial para ser utilizados en la investigación hidrológica.

Al ser la precipitación una variable clave en el balance hidrológico, con un rol dominante en el clima y tiempo, es fundamental una mejor comprensión de su distribución espacial y temporal. Sin embargo, datos de pluviómetros se encuentran escasamente y se limitan a mediciones en un único punto, mientras que en datos de radar su cobertura es limitada por la topografía, además de una baja disponibilidad al público. Existen muchos algoritmos basados en satélites para producir estimaciones de lluvias por períodos que van desde media hora a un mes y disponibles a los dos días de las observaciones por satélite; a menudo, también, en algunas horas (Aonashi et al., 2009; Huffman et al., 2002; Huffman et al., 2001; Joyce et al., 2004; Kidd et al., 2003; Sorooshian et al., 2000; Tapiador, 2002; Turk et al., 2002. Estas nuevas tecnologías están permitiendo importantes y decisivos avances, en el contexto de la hidrología aplicada, al llevarse a la práctica la modelación hidrológica distribuida con variables hidrológicas, obtenidas a través de sensores de satélite, sobre todo en zonas con carencia de datos observados. Sin embargo, se requiere su validación con mediciones de pluviómetros y/o radares en una amplia gama de condiciones meteorológicas y regímenes climáticos. Es por ello que este trabajo tiene como objetivo evaluar la utilidad de la lluvia estimada de satélite, a través de la caracterización espacial y agregada de la lluvia, y su posterior aplicación en un modelo hidrológico distribuido en la cuenca del río Júcar.

Los sensores de satélite que detectan la radiación en longitudes de onda del infrarrojo (IR) y rango visible (VIS), se basan en que los procesos de formación de lluvia implican la existencia de gotas de nube de gran tamaño y/o de partículas de hielo en la misma, que frecuentemente se difunden hacia su cima por la temperatura de brillo; estas se correlacionan positivamente con la lluvia, por lo tanto son estimaciones indirectas que proporcionan rápida información con los sensores de múltiples satélites, necesaria para capturar el crecimiento y decadencia de nubes precipitantes. Dinku et al. (2009) indican que sus principales limitaciones están referidas al tipo de nubes y condiciones atmosféricas locales como: (1) Variación local: sistemas multicapa de nubes pueden bloquear la vista de la lluvia subyacente. Además, la relación entre la temperatura en el tope de la nube y la lluvia es altamente dependiente de la estación y el lugar; (2) Efecto de lluvia cálida: regiones cerca de la costa o en zonas de montaña pueden experimentar lluvias de nubes que no llegan lo suficientemente alto en la atmósfera para registrarse como nubes frías; (3) Efecto de nubes cirros: son nubes lo suficientemente altas en la atmósfera, compuestas de cristales de hielo y que el satélite detecta como muy frías y por tanto se asocia con presencia de lluvia, aunque en realidad no la producen. Estos sensores están instalados en satélites geoestacionarios que giran sincrónicamente con la rotación de la tierra en órbitas a $36.000 \mathrm{~km}$ de altura y periodo orbital de 24 horas, por eso siempre están sobre un mismo punto sobre el Ecuador. Estos satélites ofrecen cobertura de un área limitada, pero con alta resolución temporal. Los satélites más conocidos son: GOES "Geostationary Operational Environmental Satellite", lanzados por los Estados Unidos y administrados por la NOAA "National Oceanic and Atmospheric Administration"; METEOSAT, lanzados por la Unión Europea y administrados por EUMETSAT "European Organisation for the Exploitation of Meteorological Satellites"; GMS "Geosynchronous Meteorological Satellite", lanzados por Japón.

Los sensores que operan en el espectro de las microondas (radiómetros), la radiación que las nubes y lluvia emiten, absorben y dispersan (interactúan directamente con la lluvia) reflejan el contenido total del agua integrada en la vertical, por lo que es una estimación con más base física. Estos sensores pueden medir la emisión neta térmica que emana de la parte superior de la atmósfera. $\mathrm{Su}$ principal limitación es que tienen baja resolución temporal y dificultad en la diferenciación de la señal procedente de la lluvia o de otros tipos de superficies y coberturas superficiales que tengan espectros similares. Están instalados en satélites de órbita polar a 700-800 $\mathrm{km}$ de altura pasando casi directamente sobre los polos, tardan aproximadamente 100 minutos en completar una órbita. Las imágenes son de mayor precisión y resolución espacial ya que el satélite está en una órbita mucho más baja que los geosincrónicos, pero sólo con una o dos observaciones de la misma región el mismo día (baja resolución temporal). Los satélites más conocidos son: DMSP "Defense Meteorological Satellite Program", lanzados por los Estados Unidos y administrados por la NOAA; MetOp, lanzados por la Unión Europea y administrados por EUMETSAT.

Una variante de la órbita polar es la heliosincrónica en la cual los parámetros de movimiento del satélite están calculados de forma que la superficie terrestre barrida por el mismo se encuentre siempre bajo la misma iluminación solar; es decir, las posiciones relativas del satélite y del Sol se mantienen constantes, con lo cual se consigue que las imágenes del espectro se den siempre en las mismas condiciones. El satélite más conocido es el llamado "Aqua" que es parte del grupo de satélites del EOS "Earth Observing System", lanzado por los Estados Unidos y administrado por la NOAA.

En la actualidad, para beneficiarse de las fortalezas de los diferentes tipos de sensores de satélites y minimizar los impactos de sus limitaciones, se usan técnicas mixtas que combinan la mejor lluvia identificada por sensores de 
microondas con la mayor frecuencia espacio temporal de sensores de imágenes infrarrojo (Sorooshian et al., 2002; Dinku et al., 2009).

Existen muchos algoritmos basados en satélites para estimar la lluvia a escala global, destaca el trabajo de Sorooshian et al. (2005) del Centro para la Hidrometeorología y Sensores Remotos (CHRS) con el algoritmo PERSIANN. Este algoritmo es un sistema de estimación de precipitación multi-plataforma que utiliza una tecnología de redes neuronales artificiales para combinar alta calidad con baja densidad de muestreo de datos de la NASA, la NOAA y DMSP, satélites orbitales-polares de baja altitud (TRMM, DMSP F-13, F-14 y F-15, NOAA-15, -16, -17) con datos de muestreo continuo de satélites geoestacionarios (GOES). Los datos de precipitación generados a partir de PERSIANN cubren de $50^{\circ} \mathrm{S}-50^{\circ} \mathrm{N}$, con una resolución espacial de $0,25^{\circ}$ y una resolución temporal de seis horas. Un esquema del flujo que genera el algoritmo PERSIANN se visualiza en la Fig. 1.

PERSIANN está disponible a través de la interfazHyDIS en la dirección web http://hydis8.eng.uci.edu/hydis-unesco/. En la mejora del algoritmo PERSIANN, Hong et al. (2004) desarrollaron un sistema de clasificación de nubes PERSIANN-CCS que utiliza técnicas de procesamiento de imágenes por ordenador y técnicas de reconocimiento de patrones para procesar imágenes de nubes y obtener intensidad de precipitación. Diversos estudios se han realizado para validar datos de precipitación PERSIANN para diferentes escalas hidrológicas y mesoclimas, como por ejemplo: (1) Validación de precipitación diaria y patrones de precipitaciones diurnas, con observaciones de campo proporcionadas por el TRMM (Hong et al., 2005; Sorooshian et al., 2002). (2) Validación de datos PERSIANN con precipitación diaria disponible del INMET y base de datos CPTEC sobre Sudamérica (Goncalves et al., 2006). (3) Validación de datos de precipitación por satélite con observaciones de campo de cuatro cuencas en Sudáfrica (Hughes, 2006). (4) Evaluación de las estimaciones del modelo numérico de pronóstico del tiempo MM5 sobre el suroeste de EE.UU y México (Gochis et al., 2002). (5) Asimilación de datos PERSIANN a un Sistema de Modelado Atmosférico Regional (RAMS) para investigar los procesos hidrológicos en la superficie de la tierra (Li et al., 2003; Yi, 2002; Yucel et al., 2002). (6) Validación de estimaciones de precipitación por satélite de diferentes algoritmos, entre ellos el PERSIANN, sobre Australia, USA, Europa Occidental, Japón y Sudamérica; iniciado en el 2003 por el International Precipitation Working Group. (7) Unificación de datos de pluviómetros y sistema PERSIANN sobre la región de México (Guevara, 2002). (8) Investigación de impactos de la asimilación de estimaciones de precipitación por satélite en la previsión de lluvia en el suroeste de Estados Unidos (Xu et al., 2004). (9) Análisis múltiple de los productos de precipitación y evaluación de su impacto en el Global Land Data Assimilation System (GLDAS) (Gottschalck et al., 2005).

Una recopilación de los trabajos realizados con el algoritmo PERSIANN se presenta en el capítulo SatelliteBased Precipitation Measurement Using PERSIANN System del libro Hydrological Modeling and the Water Cycle (Hsu y Sorooshian, 2008).

En la última década, se han intensificado publicaciones sobre información de precipitación obtenida de satélite a escala global, aplicada en simulación hidrológica que se detalla a continuación: (1) Simulación del caudal en el río Senegal con datos de lluvia de satélite METEOSAT y del modelo hidrológico distribuido MIKE SHE (Andersen et al., 2002). (2) Simulación del caudal de la cuenca Leaf del río Misisipi. Se empleó más de tres años de precipitación PERSIANN y datos de radar con información de pluviómetros. Se usó el modelo hidrológico conceptual SSMAM (Hsu et al., 2002). (3) Utilización de estimaciones de precipitación diaria derivados de imágenes de METEOSAT, en la cuenca Bakoye en Mali, África. El modelo hidrológico empleado es el PITMAN (Grimes y Diop, 2003). (4) Utilización del modelo hidrológico semidistribuido conceptual HBV IWS, con datos de precipitación de CCPG-NCEP y de temperatura de la NASA-GISTEM. La zona de estudio fue la cuenca del río Neckar en Alemania y la cuenca del Chirchik en Uzbekistan (Bardossy et al., 2006). (5)

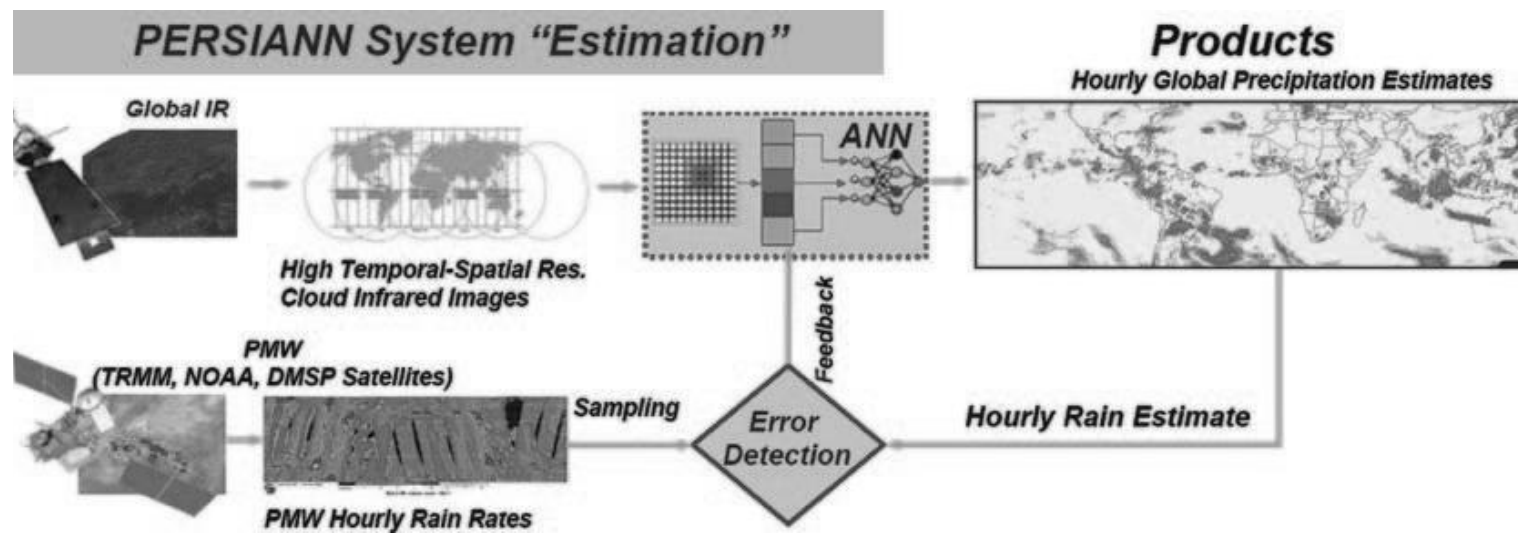

Figura 1. Flujo que genera el algoritmo PERSIANN 
Simulación del caudal en la cuenca Leaf del río Misisipi mediante el modelo hidrológico conceptual HyMOD. Se empleó datos de precipitación PERSIANN-CCS del 2002 y 2003 (Moradkhani et al., 2006). (6) Monitoreo de inundaciones por simulación del caudal de la cuenca Limpopo en Mozambique, a partir de datos de precipitación medidos por satélites NOAA-CPC y NASA-TRMM, con modelo hidrológico semidistribuido GeoSFM y datos de precipitación de 1998 a 2003 (Asante et al., 2007). (7) Empleo del modelo hidrológico distribuido MIKE SHE con información de satélite de METEOSAT-7 y sensores AVHRR, en la cuenca del río Senegal (Stisen et al., 2008). (8) Previsión de inundaciones por lluvias convectivas, usando datos de satélites GMS-5 en la cuenca del río Klang en Malasia. Se utilizó el modelo lluvia-escorrentía del hidrograma unitario, con información del año 2003 al 2006 (Wardah et al., 2008). (9) Empleo del modelo hidrológico distribuido determinístico WaSiM-ETH en la cuenca del río Volta. La información de satélite de precipitación se obtuvo de MM5 y e TRMM (Wagner et al., 2009). 10) Empleo del modelo semidistribuido mejorado SAC-SMA, que separa la componente de escorrentía rápida de la lenta, en la cuenca del río Illinois. Plantea cuatro estrategias de calibración para aprovechar las ventajas de los algoritmos de optimización SCE-UA y los sistemas MACS. Se empleó datos de precipitación horaria de once años del NOAA`s multisensor (Khakbaz et al., 2009).

\section{Materiales y métodos}

\section{Zona de estudio}

El área de estudio es la cuenca mediterránea del río Júcar con un área de $21.434 \mathrm{~km}^{2}$. La información hidrometeorológica local proviene de estaciones del SAIH y AEMET (Fig. 1) e incluye series temporales de lluvia, caudales, información de embalses y temperatura. La evapotranspiración de referencia se obtuvo con la ecuación de Hargreaves (Allen et al., 2006) previamente calibrada con información de Penman-Monteith.

\section{Metodología}

Con ayuda del servidor HyDIS se delimitó, en el mapa interactivo, las coordenadas de la cuenca del Júcar y se recortó la información del producto PERSIANN a un área rectangular cercana a la cuenca, con geometría de 11 columnas por 10 filas para un periodo desde el $1^{\circ}$ de marzo del 2000 al 31 de octubre del 2009, a una resolución temporal diaria y resolución espacial de $0,25^{\circ}$. Finalmente, se obtuvieron 3.532 archivos GRID ASCII, uno por día.

Se empleó el modelo hidrológico distribuido TETIS con parámetros efectivos que permiten corregir errores que generan incertidumbre en la modelación. Estos parámetros efectivos se ajustan a través de factores correctores que se pueden calibrar automáticamente con el algoritmo de optimización SCE-UA "Shuffled Complex Evolution".
Es así que la principal virtud del modelo TETIS es la representación explicita de la variabilidad espacial de las características físicas y el uso de una estructura separada de parámetros para la calibración de parámetros efectivos (Francés et al., 2007).

Por lo tanto, los mapas de los parámetros hidrológicos de la cuenca, se terminaron de calibrar con el modelo hidrológico mediante un factor corrector, con el que cambiarán los valores medios, pero mantendrán su estructura espacial y su coeficiente de variación. La implementación del modelo requirió información espacial de la cuenca en formato GRID ASCII como son: modelo de elevación digital, mapa de celdas acumuladas, mapa de direcciones de flujo, mapa de pendientes, mapa de velocidad en ladera, mapa de cobertura vegetal y mapas de parámetros hidrológicos. Los parámetros geomorfológicos del cauce ingresaron como coeficientes o exponentes en ecuaciones geomorfológicas de tipo potencial.

Se empleó el modelo TETIS a escala temporal diaria y escala espacial que corresponde a un tamaño de celda de $500 \mathrm{~m}$ x $500 \mathrm{~m}$. La información PERSIANN requirió una codificación para ajustarla al formato compatible con el modelo hidrológico. Es así que la información ingresa al modelo respetando la variabilidad espacial, en formato ASCII, e interpretada como estaciones virtuales localizadas en el centro de cada celda de la malla del producto del satélite: malla de 11 columnas x 10 filas que resultaron en 110 estaciones virtuales.

En la calibración automática, TETIS emplea el algoritmo SCE-UA para obtener los nueve factores correctores del modelo. Sin embargo, se pueden obtener múltiples conjuntos de parámetros que producen simulaciones aceptables con igual función objetivo, en lo que se denomina equifinalidad. Para reducir la posibilidad de caer en el problema de la equifinalidad, es necesario

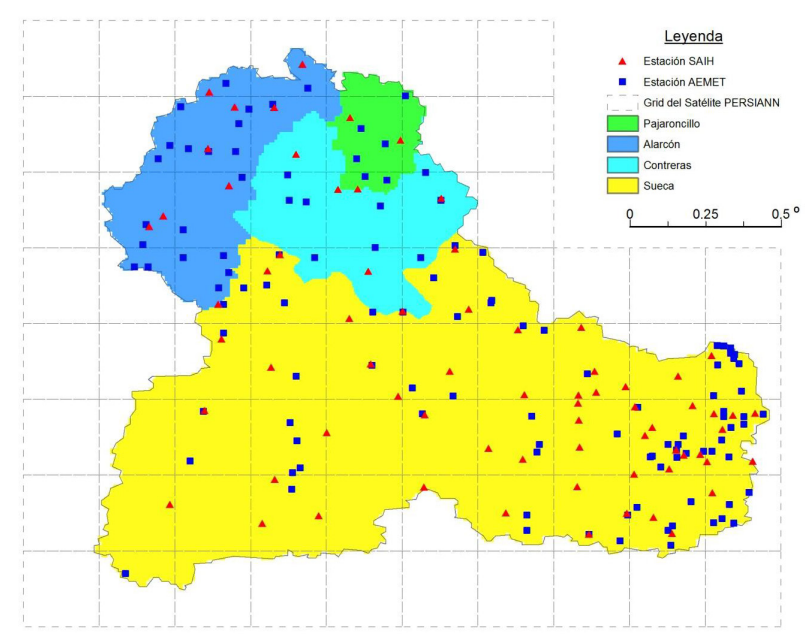

Figura 2. Distribución de información PERSIANN e información local (SAIH, AEMET) en toda la cuenca del río Júcar 
realizar una calibración manual antes que la calibración automática, que adicionalmente redundará en un menor número de iteraciones que convergen hacia la solución (lo cual tiene repercusión directa en el tiempo de cómputo de la optimización). El éxito de la calibración manual depende de la experiencia del modelador y de su conocimiento e interacción con el modelo. Por ello, se recomienda ajustar manualmente primero el flujo base y luego el porcentaje de error en el volumen, que son más sensibles a las condiciones iniciales de humedad en el tanque estático y nivel de acuífero y dejar el ajuste de los caudales máximos para la calibración automática. En la calibración automática, se tomó como función objetivo de la optimización el índice Nash-Sutcliffe (E), que es más sensible a los caudales máximos. En la validación, se usaron los factores correctores obtenidos en la calibración y para la validación temporal, se simuló la respuesta del sistema utilizando una parte de los datos de la serie temporal que no hayan sido utilizados en la calibración. Para detalles sobre el marco conceptual y el procedimiento de modelación ver Francés et al. (2007) y Vélez et al. (2009).

En la caracterización y comparación de la lluvia local y satélite, se analizaron dos casos: lluvia distribuida y lluvia agregada. En el caso de la lluvia distribuida es necesario que los datos estén en la misma escala espacial, por tanto es necesario interpolar; para ello, existen diversas herramientas geoestadísticas. Para el estudio, como la interpolación, se está usando con fines de comparación de la lluvia local y la lluvia de satélite la interpolación kriging (variograma esférico y exponencial) y la interpolación inversa de la distancia al cuadrado ya que en la modelación hidrológica, TETIS usa esta técnica para interpolar la lluvia. Se eligió como periodo a analizar desde el $1^{\circ}$ de julio del 2002 al 31 de julio del 2003 porque es el periodo de calibración que se usó en la modelación hidrológica. Los mapas mensuales se obtuvieron por sumatoria de los mapas diarios interpolados y el mapa anual se obtuvo por sumatoria de los mapas mensuales.
El criterio empleado para hacer la comparación fue la correlación lineal de Pearson (r) que es el cociente de la covarianza dividida por el producto de sus desviaciones

estándar, así: $r=\frac{\sigma_{x y}}{\sigma_{x} \sigma_{y}}$; Donde: $\sigma_{x y}$ covarianza de "x" e " $y$ "; $\sigma_{x}$ desviación estándar de " $x$ ". Las variables estadísticas se obtuvieron con la herramienta "Band collection statistics" del ArcGIS. Esta herramienta proporciona el análisis multivariable de un conjunto de rasters y calcula la matriz de correlación de Pearson así como parámetros estadísticos básicos de cada raster.

En el caso de la lluvia agregada, esta se analizó a la salida de las subcuencas de Pajaroncillo, Contreras, Alarcón y Sueca, información que se obtiene del modelo TETIS que permite obtener la lluvia agregada real en cualquier punto de la cuenca. Se analizó información diaria desde el $1^{\circ}$ de marzo del 2000 al 31 de octubre del 2009. El criterio empleado para hacer la comparación fue la correlación lineal de Pearson (r).

\section{Resultados y discusión}

\section{Caracterización de la lluvia}

Del mapa de lluvia distribuida anual (Fig. 3), se obtiene con PERSIANN valores más altos de lluvia en la zona cercana al mediterráneo y valores más bajos en la zona montañosa del sistema ibérico. Esto induce a creer que el satélite representa mejor las lluvias ocurridas por el fenómeno meteorológico "gota fría" que ocurre en la parte baja de la cuenca. Al contrario de lo que ocurre en la parte alta, en que los datos de satélite no estarían representando bien el efecto de las cadenas de montañas que emiten radiación muy variable, en comparación con la superficie del mar, que es más homogénea.

Del mapa de lluvia distribuida mensual y diaria (Figs. 4 y 5), la correlación espacial mejora, sobre todo en la

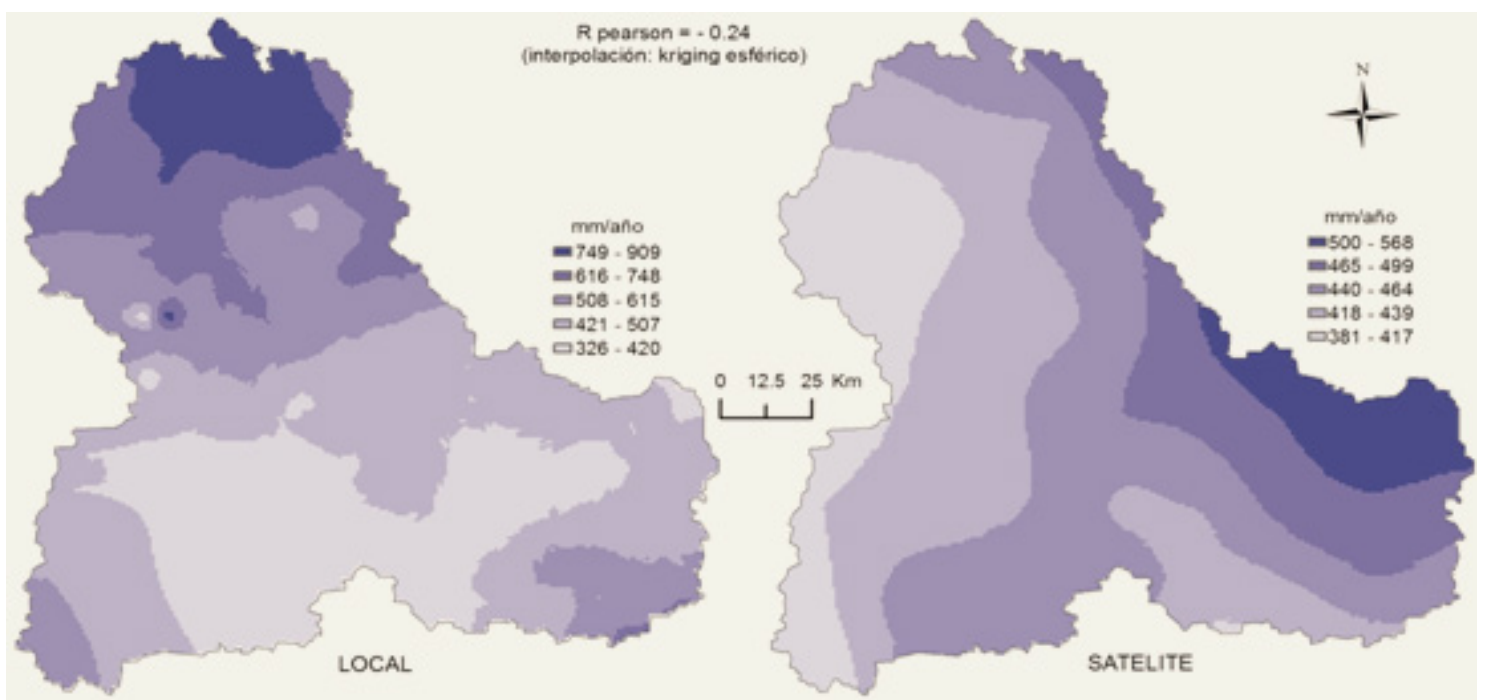

Figura 3. Mapa de lluvia distribuida anual entre lluvia local y lluvia de satélite. Periodo analizado: 

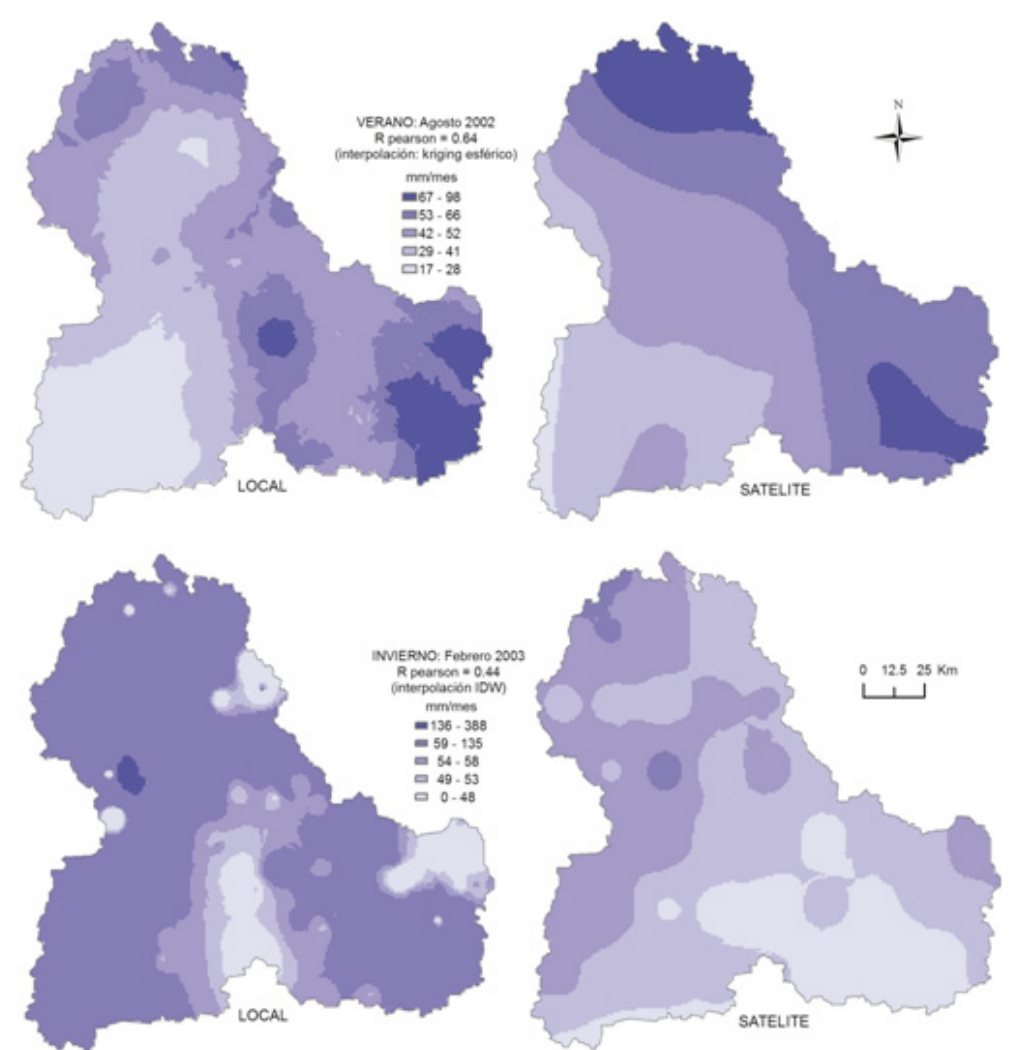

Figura 4. Mapa de lluvia distribuida mensual de mejor correlación entre lluvia local y lluvia de satélite. Periodo analizado: Julio 2002 a Julio 2003
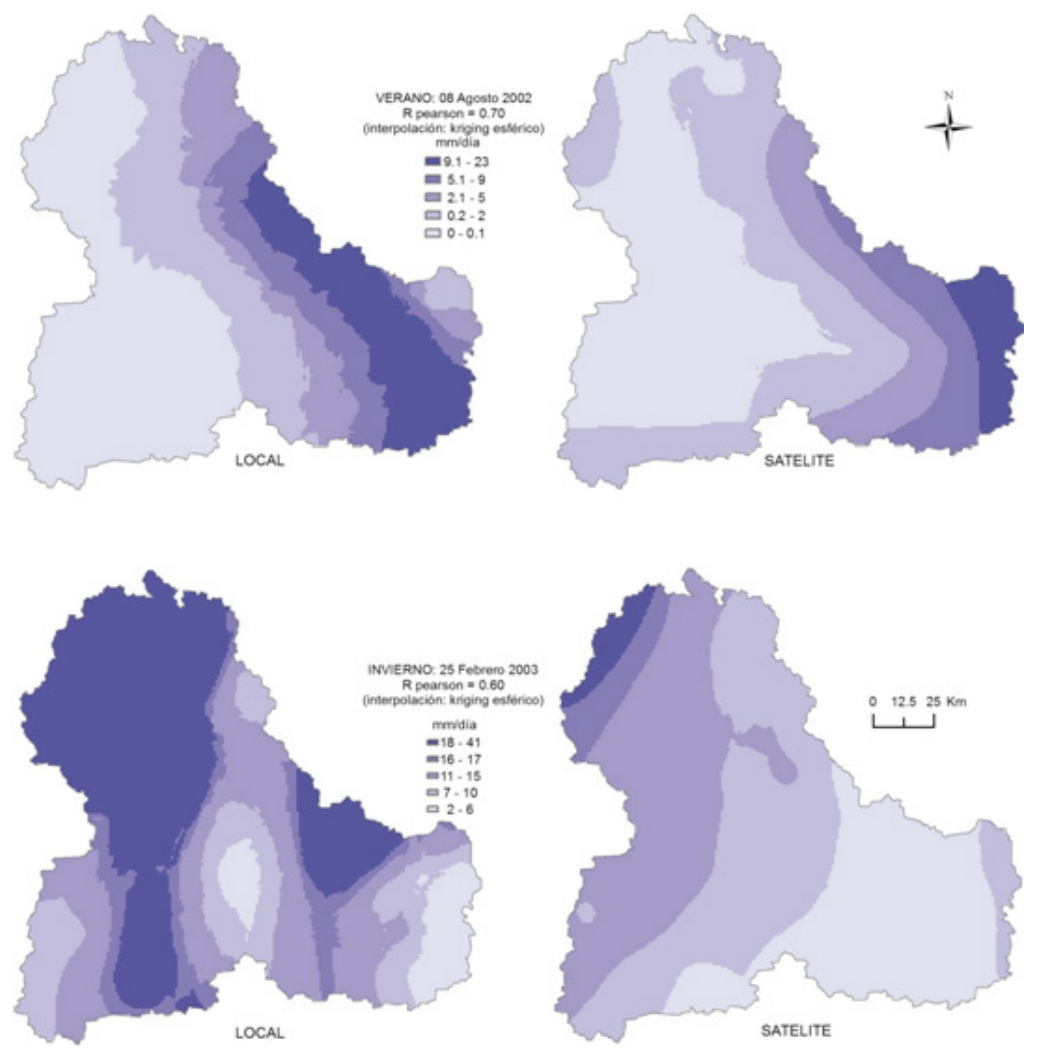

Figura 5. Mapa de lluvia distribuida diaria de mejor correlación entre lluvia local y lluvia de satélite. Periodo analizado: $1^{\circ}$ de Julio del 2002 al 31 de Julio del 2003 
estación de verano. Se visualiza que en este periodo los valores más altos diarios $(23 \mathrm{~mm} /$ día $)$ se concentran en la parte baja y media de la cuenca, esto debido a la alta evaporación por las mayores temperaturas en el agua del mar y por consiguiente mayor humedad en las zonas cercanas al litoral. En cambio, en invierno los valores más altos $(41 \mathrm{~mm} /$ día $)$ se concentran en la parte alta y media de la cuenca originada por la orografía de la cadena de montañas del sistema ibérico.

Respecto a la lluvia agregada diaria a la salida de las subcuencas de Pajaroncillo, Contreras, Alarcón y Sueca (Fig. 6 y Tabla 1), se observa que el valor promedio es significativamente menor con lluvia satélite $(0,91$ a 1,02 $\mathrm{mm} / \mathrm{d})$ que con lluvia local $(1,27$ a $1,69 \mathrm{~mm} / \mathrm{d})$, con correlaciones de Pearson que varían de 0,44 a 0,48.

\section{Modelación hidrológica}

La calibración y validación con precipitación del producto PERSIANN se realizó a la salida de las subcuencas Pajaroncillo y Sueca, obteniéndose resultados prometedores con valores del índice de Nash-Sutcliffe, el error en el volumen y el error cuadrático medio RMSE que se detallan en la Tabla 2 y se visualizan en los hidrogramas de las Figs. 7 y 8.

\section{Modelación hidrológica}

La calibración y validación con precipitación del producto PERSIANN se realizó a la salida de las subcuencas Pajaroncillo y Sueca, obteniéndose resultados prometedores con valores del índice de Nash-Sutcliffe, el error en el volumen y el error cuadrático medio RMSE que se detallan en la Tabla 2 y se visualizan en los hidrogramas de las Figs. 7 y 8.

Aunque las observaciones por satélite son capaces de proporcionar la distribución espacial y temporal de las precipitaciones, las mediciones tienden a mostrar un sesgo sistemático debido a que son estimaciones indirectas que dependen de las propiedades de la nube (imagen infrarroja) o del líquido contenido en las nubes y el hielo (imágenes de microondas pasivo). Quizás la relación asumida por el producto PERSIANN entre la temperatura en el tope de nube y la intensidad de la precipitación, no es la más adecuada para esta región mediterránea del Júcar, que se caracteriza por lluvias de muy alta intensidad y corta duración de origen convectivo.

\section{Conclusión}

Los actuales modelos hidrológicos distribuidos permiten simular caudales no únicamente en la salida de una cuenca,

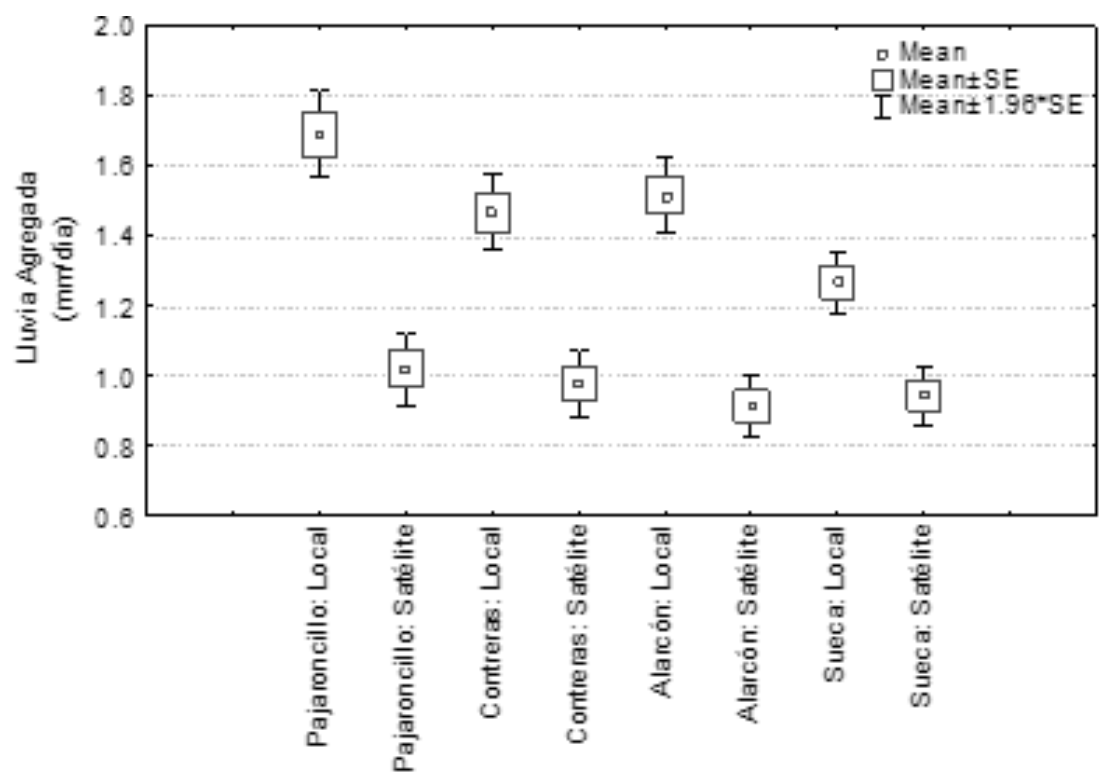

Figura 6. Lluvia agregada diaria local y satélite

Tabla 1. Resumen de estadísticos de lluvia agregada diaria local y satélite

\begin{tabular}{|l|r|r|r|r|}
\hline Detalle & Pajaroncillo & Contreras & Alarcón & Sueca \\
\hline Área drenada $\left(\mathrm{km}^{2}\right)$ & 861 & 3427 & 2883 & 21523 \\
\hline Altitud $(\mathrm{msnm})$ & 1009 & 630 & 799 & 0 \\
\hline $\mathrm{N}^{\text {o de datos }}$ & 3332 & 332 & 332 & 332 \\
\hline Lluvia promedio local & 1,69 & 1,47 & 1,51 & 1,27 \\
\hline satélite & 1,02 & 0,98 & 0,91 & 0,94 \\
\hline Lluvia máxima local & 38,67 & 32,33 & 24,75 & 34,01 \\
\hline \multicolumn{1}{|c|}{ satélite } & 36,75 & 36,35 & 34,48 & 34,12 \\
\hline R Pearson & 0,47 & 0,48 & 0,44 & 0,47 \\
\hline
\end{tabular}


Tabla 2. Resultados de calibración y validación con el producto de satélite PERSIANN

\begin{tabular}{|c|c|c|c|c|}
\hline \multirow[b]{2}{*}{ Principales Características } & \multicolumn{2}{|c|}{ Pajaroncillo } & \multicolumn{2}{|r|}{ Sueca } \\
\hline & $\begin{array}{l}\text { Calibración } \\
\text { Jul02-Jul03 }\end{array}$ & $\begin{array}{l}\text { Validación Temporal } \\
\text { Mar00-Oct09 }\end{array}$ & $\begin{array}{l}\text { Calibración } \\
\text { Jul02-Jul03 }\end{array}$ & $\begin{array}{c}\text { Validación Temporal } \\
\text { Mar00-Oct09 }\end{array}$ \\
\hline Caudal máx observado (m3/s) & 54,075 & 64,720 & 361,095 & 639,345 \\
\hline Caudal máx simulado (m3/s) & 28,394 & 41,950 & 275,226 & 616,055 \\
\hline Nro de datos & 396,000 & 3511,000 & 396,000 & 3532,000 \\
\hline Caudal medio observado & 5,964 & 3.391 & 90,568 & 84,675 \\
\hline Volumen Observado (Hm3) & 204,038 & 1028,725 & 3098,745 & 25839,868 \\
\hline Volumen Simulado (Hm3) & 186,357 & 1233,286 & 2664,485 & 16367,897 \\
\hline Error en Volumen(\%) & $-8,666$ & 19,885 & -14.014 & $-36,656$ \\
\hline Índice de Nash-Sutcliffe & 0,384 & $-0,714$ & 0,499 & $-0,150$ \\
\hline $\operatorname{RMSE}(\mathrm{m} 3 / \mathrm{s})$ & 4,647 & 5,779 & 40,599 & 71,236 \\
\hline
\end{tabular}

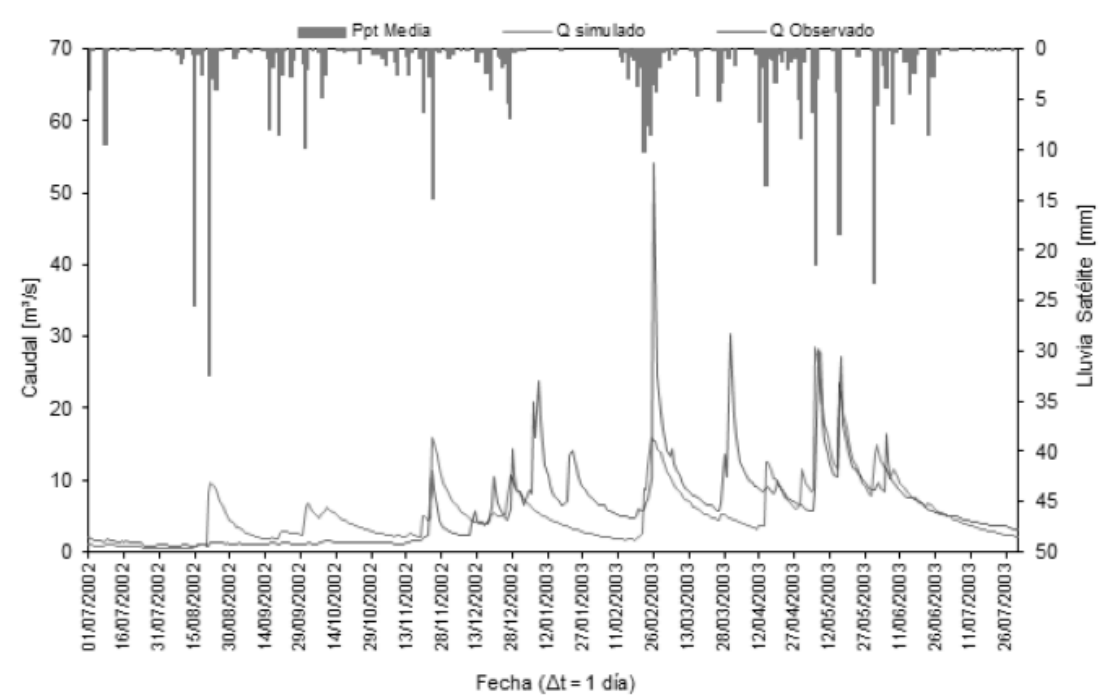

Figura 7. Hidrograma de caudal observado y simulado con lluvia PERSIANN a la salida de la subcuenca Pajaroncillo. Periodo analizado: $1^{\circ}$ Julio 2002 - 31 Julio 2003

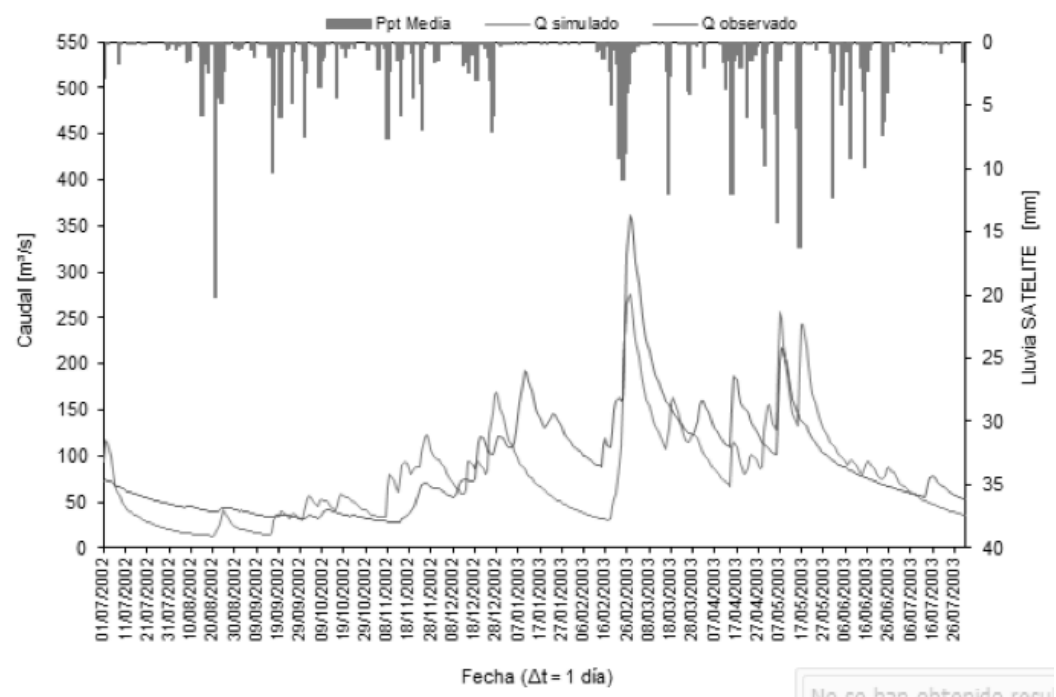

Figura 8. Hidrograma de caudal observado y simulado con lluvia PERSIANN a la salida de la subcuenca Sueca. Periodo analizado: 01 Julio 2002 - 31 Julio 2003 
sino en cualquier parte de la misma, pero la eficacia de estos modelos depende de la disponibilidad de los datos de entrada. Es así que la lluvia estimada de satélite a escala global se adapta a estos modelos distribuidos ya que se tienen datos de lluvia para toda la cuenca. Sin embargo, debido a la multidimensionalidad del error de la lluvia estimada de satélite, es difícil establecer a priori un producto que permita una óptima aplicación hidrológica en diferentes condiciones climáticas y es por eso que se hace necesario evaluar su desempeño a través de la modelación hidrológica. En este estudio, se evaluó la utilidad de la lluvia estimada por satélite a través de un modelo hidrológico lluvia-escorrentía y se empleó la lluvia estimada por el algoritmo PERSIANN a una resolución temporal diaria y resolución espacial de $0,25^{\circ}$ para el periodo comprendido entre el $1^{\circ}$ de marzo del 2000 al 31 de octubre del 2009 en la cuenca del río Júcar (España), obteniéndose resultados prometedores. Resulta el mejor rendimiento del modelo en calibración con valores de 0,384 y 0,499 del índice de Nash-Sutcliffe a la salida de las subcuencas Pajaroncillo y Sueca.

Los pluviómetros y estimaciones de satélite tienen errores de diferente naturaleza: las mediciones pluviométricas tienden a ser más precisas en un punto mientras que su significado espacial decae con la distancia y por lo tanto con el área; en cambio las estimaciones por satélite ofrecen una mejor representación espacial (aunque sesgado). Por ello, la tendencia actual es el uso de datos de satélite combinados con los mejores datos disponibles, independientemente de la fuente. Con el avance de la ciencia llegarán nuevos sensores de satélite y tecnologías que mejorarán la detección y su utilidad en la modelación hidrológica.

\section{Literatura citada}

Allen, R.; Pereira, L.; Raes, D. y Smith, M. 2006. Evapotranspiración del cultivo: Guías para la determinación de los requerimientos de agua de los cultivos. Roma, Italia: FAO.

Andersen, J.; Dybkjaer, G.; Jensen, K.H. 2002. Use of remotely sensed precipitation and leaf area index in a distributed hydrological model. Journal of Hydrology, 264: $34-50$.

Aonashi, K.; Awaka, J. e Hirose, M. 2009. GSMaP passive microwave precipitation retrieval algorithm: Algorithm description and validation. Journal of the Meteorological Society of Japan, 87A:119-136.

Asante, K.O.; Macuacua, R.D. y Artan, G.A. 2007. Developing a flood monitoring system from remotely sensed data for the Limpopo Basin. IEEE Transactions on Geoscience and Remote Sensing, 45 (6): 1709-1714.

Bardossy, A.; Gafurov, A. y Gotzinger, J. 2006. Hydrological modelling for meso-scale catchments using globally available data. Hydrology and Earth System Sciences, 4: 2209-2242.

Biftu, G.F. y Gan, T.Y. 2001. Semi-distributed, physically based, hydrologic modelling of the Paddle River Basin, Alberta, using remotely sensed data. Journal of Hydrology, 244(3-4): 137-156.

Boegh, E.; Thorsen, M. y Butts, M.B. 2004. Incorporating remote sensing data in physically based distributed agro-hydrological modelling. Journal of Hydrology, 287 (1-4): 279-299.

Chen, J.M.; Chen, X.Y. y Ju, W.M. 2005. Distributed hydrological model for mapping evapotranspiration using remote sensing inputs. Journal of Hydrology, 305 (1-4): 15-39.

Dinku, T.; Funk, C. y Grimes, D. 2009. The potential of satellite rainfall estimates for index insurance. International Research Institute for Climate and Society (IRI). The Earth Institute at Columbia University.

Engman, E.T. 1995. Recent advances in remote sensing in hydrology. Reviews of Geophysics. American Geophysical Union.

Francés, F.; Vélez, J.I. y Vélez, J.J. 2007. Splitparameter structure for the automatic calibration of distributed hydrological models. Journal of Hydrology, 332: 226-240.

Gochis, D. J.; Shuttleworth, W.J. y Yang, Z.L. 2002. Sensitivity of the modelled North American Monsoon regional climate to convective parameterization. Monthly Weather Review, 130: 1282-1298.

Goncalves, G.L.; Shuttleworth, W.J. y Nijssen, B. 2006. Evaluation of model-derived and remotely sensed precipitation products for continental South America. Journal of Geophysical Research. 111 D161113.

Gottschalck, J.; Meng, J. y Rodell, M. 2005. Analysis of multiple precipitation products and preliminary assessment of their impact on Global Land Data Assimilation System (GLDAS) land surface states. Journal of Hydrometeorology, 6 (5): 573-598.

Grimes, D.I.F. y Diop, M. 2003. Satellite-based rainfall estimation for river flow forecasting in Africa. I: Rainfall estimates and hydrological forecasts. Hydrological Sciences Journal des Sciences Hydrologiques, 48 (4): 567-584.

Guevara, J.M. 2002. Precipitation estimation over Mexico applying PERSIANN system and gauge data. (Master Thesis). University of Arizona.

Hansen, J.E.; Ruedy, R. y Sato, M. 2009. NASA GISS Surface Temperature (GISTEMP) Analysis. In Trends: A Compendium of Data on Global Change. Carbon Dioxide Information Analysis Center, Oak Ridge National Laboratory, U.S. Department of Energy, Oak Ridge, Ten n., U.S.A.: Center for Climate Systems Research, NASA Goddard Institute for Space Studies.

Hong, Y.; Hsu, K. y Gao, X. 2004. Precipitation estimation from remotely sensed imagery using artificial neural network-cloud classification system. Journal of Applied Meteorology, 43(12): 1834-1853.

Hong, Y.; Hsu, K.y Sorooshian, S. 2005. Enhanced 
signal of diurnal variability of rainfall retrieval from TRMM-adjusted PERSIANN algorithm. Journal of Geophysical Research, 110 - D06102.

Hsu, K.; Soroosh, S. y Gupta, H.V. 2002. Hydrologic Modelling and Analysis Using a Self-Organizing Linear Output Network. Switzerland: The International Environmental Modelling \& Software Society i. iEMSs.

Hsu, K. y Sorooshian, S. 2008. Satellite-Based Precipitation Measurement Using PERSIANN System. In: Sorooshian, S., Hsu, K., Coppola, E. (Editors), Hydrological Modelling and the Water Cycle. Water Science and Technology Library.

Huffman, G.J.; Adler, R.F. y Morrissey, M.M. 2001. Global precipitation at one-degree daily resolution from multisatellite observations. Journal of Hydrometeorology, 2: $36-50$.

Huffman, G.J.; Adler, R.F. y Stocker, E.F. 2002. $A$ TRMM-based system for real-time quasi-global merged precipitation estimates.

Hughes, D.A. 2006. Comparison of satellite rainfall data with observations from gauging station networks. Journal of Hydrology, 327: 399-410.

Joyce, R.J., Janowiak, J.E. y Arkin, P.A. 2004. CMORPH: A Method that produces global precipitation estimates from passive microwave and infrared data at high spatial and temporal resolution. Journal of Hydrometeorology, 5: 487-503.

Khakbaz, B.; Imam, B. y Hsu, K. 2009. From lumped to distribute via semi-distributed via semi-distributed: Calibration strategies for semi-distributed hydrologic models. Journal of Hydrology.

Kidd, C.; Kniveton, D.R. y Todd, M.C. 2003. Satellite rainfall estimation using combined passive microwave and infrared algorithms. Journal of Hydrometeorology, 4(1088): 1104.

Li, J.; Gao, X. y Maddox, R.A. 2003. Summer weather simulation for the semi-arid lower Colorado River basin. Monthly Weather Review, 131(3): 521-541.

Moradkhani, H.; Hsu, K. y Hong, Y. 2006. Investigating the impact of remotely sensed precipitation and hydrologic model uncertainties on the ensemble streamflow forecasting. Geophysical Research Letters, 33, L12401.

Sorooshian, S.; Gao, X. y Hsu, K. 2002. Diurnal variability of tropical rainfall retrieved from combined GOES and TRM satellite information. Journal of Climate, 15: 983-1001.

Sorooshian, S.; Hsu, K. y Bisher, I. 2005. Global Precipitation Estimation from Satellite Image using Artificial Neural Networks. International G-WADI Modelling Workshop. India: National Institute of Hydrology.
Sorooshian, S.; Hsu, K. y Gao, X. 2000. Evaluation of PERSIANN system satellite-based estimates of tropical rainfall. Bulletin of American Meteorology Society, 81: 2035-2046.

Stisen, S.; Jensen, K.H. y Sandholt, I. 2008. A remote sensing driven distributed hydrological model of the Senegal River basin. Journal of Hydrology, 354: 131148 .

Tapiador, F.J. 2002. A new algorithm to generate global rainfall rates from satellite infrared imagery. Revista de Teledetección de España. AET. 18: 57-61.

Turk, J.; Ebert, E. y Oh, H.J. 2002. Verification of an operational global precipitation analysis at short time scales. Madrid, Spain: 1st Intl. Precipitation Working Group (IPWG) Workshop.

Vélez, J.J.; Puricelli, M.; López, F. y Francés, F. 2009. Parameter extrapolation to ungauged basins with a hydrological distributed model in a regional framework. Hydrol. Earth Syst. Sci, 13: 229-246.

Wagner, S.; Kunstmann, H. y Bárdossy, A. 2009. Water balance estimation of a poorly gauged catchment in West Africa using dynamically downscaled meteorological fields and remote sensing information. Physics and Chemistry of the Earth, 34: 225-235.

Wardah, T.; Bakar, S.H. y Bardossy, A. 2008. Use of geostationary meteorological satellite images in convective rain estimation for flash-flood forecasting. Journal of Hydrology, 356: 283-298.

Xu, J.; Gao, X. y Sorooshian, S. 2004. Impact of radar derived and satellite derived rainfall assimilation on the rainfall predict in the southwest United States. Tollus.

Yi, H. 2002. Assimilation of satellite-derived precipitation into the regional atmospheric modelling system (RAMS) and its impact on the weather and hydrology in the Southwest United States. (Ph.D. Dissertation). Department of Hydrologic and Water Resources, University of Arizona.

Yucel, I.; Shuttleworth, W.J. y Pinker, R.T. 2002. Impact of ingesting satellite-derived cloud into the regional atmospheric modelling system. Monthly Weather Review, 13: 610-628. 